\title{
LOCALIZATION OF QUANTUM STATES AND LANDSCAPE FUNCTIONS
}

\author{
STEFAN STEINERBERGER
}

(Communicated by Svitlana Mayboroda)

\begin{abstract}
Eigenfunctions in inhomogeneous media can have strong localization properties. Filoche and Mayboroda showed that the function $u$ solving $(-\Delta+V) u=1$ controls the behavior of eigenfunctions $(-\Delta+V) \phi=\lambda \phi$ via the inequality

$$
|\phi(x)| \leq \lambda u(x)\|\phi\|_{L^{\infty}} .
$$

This inequality has proven to be remarkably effective in predicting localization and recently Arnold, David, Jerison, Mayboroda and Filoche connected $1 / u$ to decay properties of eigenfunctions. We aim to clarify properties of the landscape: the main ingredient is a localized variation estimate obtained from writing $\phi(x)$ as an average over Brownian motion $\omega(\cdot)$ started in $x$

$$
\phi(x)=\mathbb{E}_{x}\left(\phi(\omega(t)) e^{\lambda t-\int_{0}^{t} V(\omega(z)) d z}\right) .
$$

This variation estimate will guarantee that $\phi$ has to change at least by a factor of 2 in a small ball, which implicitly creates a landscape whose relationship with $1 / u$ we discuss.
\end{abstract}

\section{INTRODUCTION}

1.1. The landscape function. It is well known that physical systems comprised of inhomogeneous materials can exhibit peculiar vibration properties: let $\Omega \in \mathbb{R}^{n}$ be open, bounded and

$$
(-\Delta+V) \phi=\lambda \phi \quad \text { in } \Omega \text { with Dirichlet boundary conditions, }
$$

where $V: \Omega \rightarrow \mathbb{R}_{\geq 0}$ is a real-valued, nonnegative potential. Anderson [2] noticed that for some potentials the low-lying eigenfunctions tend to strongly localize in a subregion of space in a very complicated manner. It seemed difficult to get any information about the localization behavior of these first few eigenfunctions without explicitly computing them.

In a remarkable contribution, Filoche \& Mayboroda [11] have given a simple but astonishingly effective method to predict the behavior of low-energy eigenfunctions. Their approach is based on the following inequality: if we associate to the problem the landscape function $u: \Omega: \mathbb{R} \rightarrow \mathbb{R}$ given as the solution of

$$
(-\Delta+V) u=1 \quad \text { in } \Omega \subset \mathbb{R}^{n} \text { with Dirichlet boundary conditions, }
$$

then there is the inequality

$$
|\phi(x)| \leq \lambda u(x)\|\phi\|_{L^{\infty}(\Omega)} .
$$

Received by the editors May 23, 2016.

2010 Mathematics Subject Classification. Primary 35P20; Secondary 82B44.

Key words and phrases. Laplacian eigenfunction, localization, torsion function, Feynman-Kac. 
The regions where $u$ is small will be of particular interest because an eigenfunction $\phi$ can only localize in $\{x: \lambda u(x) \geq 1\} \subset \Omega$. The landscape function turns out to be more effective than that: it is instructive to regard the graph of $u(x)$ as a landscape comprised of 'peaks' and 'valleys'; the valleys may then be understood as inducing a partition of the domain. Numerical experiments [11] suggest that low-lying eigenfunctions respect that partition and favor localization in one or at most a few elements in that partition. Moreover, these localized eigenfunctions are 'almost' compactly supported in the sense that in crossing from one element of the partition to another eigenfunctions seem to experience exponential decay when crossing the valley (see [11]).

1.2. The effective potential. The exponential drop in size of an eigenfunction when crossing the valley was recently studied by Arnold, David, Jerison, Mayboroda \& Filoche 3 who point out that the inverse of the landscape function $1 / u(x)$ acts as an effective potential responsible for the exponential decay of the localized states (the connection being that $u(x)$ is small in valleys, which makes $1 / u(x)$ large and large potentials induce large decay). Their approach is based on writing an eigenfunction as $\phi=u \psi$ for some unknown function $\psi$. The equation

$$
(-\Delta+V) \phi=\lambda \phi
$$

then transforms into

$$
\left[\frac{1}{u^{2}} \operatorname{div}\left(u^{2} \nabla \psi\right)\right]+\frac{1}{u} \psi=\lambda \psi .
$$

The new dominating potential $W \equiv 1 / u$ is now responsible for the underlying dynamics. The next step is to build an Agmon distance

$$
\rho\left(r_{1}, r_{2}\right)=\min _{\gamma}\left(\int_{\gamma} \sqrt{(W(r)-\lambda)_{+}} d s\right),
$$

where $\gamma$ ranges over all paths from $r_{1}$ to $r_{2}$ and use Agmon's inequality 1 to deduce that for eigenfunctions $\phi$ localized in $r_{0} \in \Omega$

$$
|\phi(r)| \lesssim e^{-\rho\left(r_{0}, r\right)}
$$

This indicates that $W \equiv 1 / u$ is playing a distinguished role. The paper [3] also gives convincing numerical evidence that $W-\lambda$ seems to predict decay more accurately than the classical quantity $V-\lambda$. This might seem surprising because $V$ determines the behavior of the eigenfunctions.

1.3. Organization. The purpose of our paper is to further clarify these observations and the interplay between an eigenfunction doubling its size in a small ball and the landscape function; the main tool is an identity following from the Feynman-Kac formula. More precisely, we

- derive and discuss the relevant identity,

- use it to prove a variation estimate localized in a small ball,

- show how w.r.t. decay $V$ is not as important as a suitable mollification of $V$,

- compare how $1 / u(x)$ fits into that framework

- and discuss some refinements of the landscape function $u(x)$. 
We always assume that $\Omega \subset \mathbb{R}^{n}$ is bounded with a smooth boundary and $V \in C^{2}(\Omega)$ to be continuous. Technically, this excludes 'block potentials' (which are only $L^{\infty}$ ) but it is clear that the first few eigenfunctions hardly change if a potential is replaced by a suitable mollification and therefore the assumption is without loss of generality.

\section{LOCAL ANALYSis OF THE HEAT FLOW}

2.1. The torision function. The landscape function arising from $V=0$, i.e., the solution of

$$
-\Delta u=1 \quad \text { in } \Omega \text { with Dirichlet boundary conditions, }
$$

is a classical object in shape optimization called the torsion function. It appears in elasticity theory [5], heat conduction [17] and geometry [14]. A version of a landscape function with potential already appeared in the context of homogenization in work of Coifman \& Meyer (unpublished, but see the application to parabolic operators by $\mathrm{S} . \mathrm{Wu}[18$ ). The most prominent role of the torsion function in the field of shape optimization (see e.g. [6]) is that $u(x)$ gives the expected lifetime of Brownian motion started in $x$ until it hits the boundary. This suggests to interpret the landscape function in that language; the idea of using Brownian motion to analyze decay properties of eigenfunctions is classical and was very successfully used in seminal papers by Carmona [7, Carmona \& Simon [8, Carmona, Masters \& Simon [9], Simon [15] and others; recently, a similar technique was used by the author [16] to obtain bounds on the size of nodal sets of Laplacian eigenfunctions $\{x: \phi(x)=0\}$ on compact manifolds.

2.2. The idea. The crucial ingredient is a simple equation representing an eigenfunction $\phi(x)$ as a localized average over local Brownian motion paths running for a short time. This equation is not new and has been used earlier for very similar purposes; see e.g. Carmona \& Simon [8]. It is obtained by looking at the effect of the semigroup $e^{t(\Delta-V)}$ on the eigenfunction. Since eigenfunctions diagonalize the semigroup, we have that if

$$
(-\Delta+V) \phi=\lambda \phi, \quad \text { then } \quad e^{t(\Delta-V)} \phi=e^{-\lambda t} \phi .
$$

At the same time, there is another interpretation of the action of the semigroup in terms of Brownian motion. The Feynman-Kac formula states that for an arbitrary function $f$

$$
e^{t(\Delta-V)} f(x)=\mathbb{E}_{x}\left(f(\omega(t)) e^{-\int_{0}^{t} V(\omega(z)) d z}\right),
$$

where the expectation $\mathbb{E}_{x}$ is taken with respect to Brownian motion $\omega(\cdot)$ started in $x$, running for time $t$ and destroyed upon impact on the boundary. Combining these two equations, we get

$$
\forall t \geq 0, \quad \phi(x)=\mathbb{E}_{x}\left(\phi(\omega(t)) e^{\lambda t-\int_{0}^{t} V(\omega(z)) d z}\right) .
$$

This equation describes a complicated relationship between $\phi(x), \lambda$ and $V$, however, it is perfectly suited for establishing a variation estimate in a small ball: assuming the eigenfunction $\phi$ to be essentially constant on a small scale allows us to move the eigenfunction $\phi$ out of the expectation at the cost of a very small error. We sketch a nonrigorous version of the argument. 


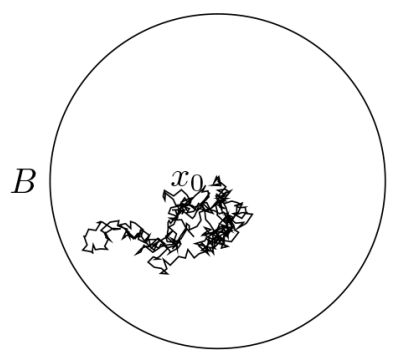

FiguRE 1. Brownian motion started in $x_{0}$.

Assume w.l.o.g. that $\phi\left(x_{0}\right)>0$. Let $B=B\left(x_{0}, r\right)$ be the ball centered at $x_{0}$ with maximal radius $r>0$ such that

$$
\forall x \in B: \frac{1}{2}\left|\phi\left(x_{0}\right)\right| \leq|\phi(x)| \leq 2\left|\phi\left(x_{0}\right)\right| .
$$

Assume furthermore that $V\left(x_{0}\right) \geq \lambda$ and that $V$ is essentially constant on $B$. We now consider the equation above for $t=c_{n} r^{2}$ with $c_{n}$ a small universal constant depending only on the dimension of $\Omega$. By making $c_{n}$ sufficiently small, we can ensure that $99 \%$ of all Brownian paths are fully contained in $B$ up to time $t$. Then

$$
\phi\left(x_{0}\right)=\mathbb{E}_{x_{0}}\left(\phi(\omega(t)) e^{\lambda t-\int_{0}^{t} V(\omega(z)) d z}\right) \sim \phi\left(x_{0}\right) \mathbb{E}_{x_{0}}\left(e^{\lambda t-\int_{0}^{t} V(\omega(z)) d z}\right) .
$$

In order for this to hold, we clearly require

$$
\mathbb{E}_{x_{0}} e^{\lambda t-\int_{0}^{t} V(\omega(z)) d z} \sim 1 \quad \text { and thus } \quad-1 \ll \lambda t-\int_{0}^{t} V(\omega(z)) d z \leq 0 .
$$

However, this last quantity can be approximated by

$$
0 \geq \lambda t-\int_{0}^{t} V(\omega(z)) d z \sim t\left(\lambda-V\left(x_{0}\right)\right) \sim r^{2}\left(\lambda-V\left(x_{0}\right)\right) \gg-1
$$

which is a statement about the maximal size of $r$ depending on $\lambda-V\left(x_{0}\right)$. We will now make this heuristic sketch precise and phrase it in classical terms. However, we emphasize that the most useful way of thinking about the setup to be in terms of path integrals: the condition $V(x)-\lambda \geq c$ could, for example, be replaced by $V(x)-\lambda \geq 0$ and $V(x)-\lambda \geq c$ true 'on average'.

Theorem 1 (Variation estimate). Suppose $(-\Delta+V) \phi=\lambda \phi$ and $V \geq 0$. There exists a universal constant $c_{n}$ (depending only on the dimension) such that the following holds: if, for some $c>0$,

$$
V(x)-\lambda \geq c \quad \text { or } \quad V(x)-\lambda \leq-c
$$

uniformly on the ball

$$
B=B\left(x_{0}, \frac{c_{n}}{\sqrt{c}} \sqrt{\frac{\lambda}{c}+\log \left(c_{n} \frac{\|\phi\|_{L^{\infty}}}{\left|\phi\left(x_{0}\right)\right|}\right)}\right) \subset \Omega,
$$

then we have

$$
\frac{\sup _{x \in B}|\phi(x)|}{\inf _{x \in B}|\phi(x)|} \geq 2
$$


The classical Harnack inequality (see e.g. [13]) for a positive solution of a second order elliptic differential equation on a domain $\Omega$ states that for $\Omega^{\prime} \subset \Omega$

$$
\sup _{\Omega^{\prime}} \phi \leq C \inf _{\Omega^{\prime}} \phi
$$

$C$ depends on the differential operator and how $\Omega^{\prime}$ is contained in $\Omega$. Our result may be seen as an opposite statement: we specify domains $\Omega^{\prime}$ on which $C$ needs to be at least of size 2. Also related in spirit are doubling estimates; see for example Bakri 4 ] or the survey of Zelditch [19]. We start by discussing the case $V(x)-\lambda \geq c$. Then the result has the expected scaling and translates into the classical $\sqrt{(V-\lambda)_{+}}$ factor in the Agmon metric. The proof of Theorem 1 yields a stronger result: the uniform estimate $V-\lambda \geq c$ is not necessary (it is only required 'w.r.t. path integrals') and we can rephrase the condition.

Local. $\phi$ varies by a constant factor on scale $\sim 1 / \sqrt{(V(x)-\lambda)_{+}}$.

Nonlocal. $\phi$ varies by a constant factor on the scale $\sim \sqrt{t_{x}}$ where

$$
t_{x}=\inf \left\{t>0: \mathbb{E}_{x} e^{\lambda t-\int_{0}^{t} V(\omega(z)) d z} \leq \frac{1}{2}\right\} .
$$

The nonlocality of the second formulation incorporates the diffusive action of the partial differential equation. Moreover, the nonlocal formulation allows for $t$ to be large. At the crudest level, the two estimates coincide because

$$
\mathbb{E}_{x} e^{\lambda t-\int_{0}^{t} V(\omega(z)) d z} \sim 1-(\lambda-V(x)) t .
$$

This estimate is only correct up to first order; one might suspect that the estimate should hold up to second order because Brownian motion is isotropic and therefore by symmetry

$$
\mathbb{E}_{x}\langle\nabla V(x), \omega(t)-x\rangle=0 .
$$

However, there is a curious contribution: Brownian motion moves to distance $\sim \sqrt{t}$ within time $t$ and this has the curious effect of turning the local geometry of $V$ at second order into a first order contribution in time

$$
\mathbb{E}_{x}\left\langle\omega(t)-x,\left(D^{2} V\right)(x) \omega(t)-x\right\rangle=t \Delta V(x) .
$$

This gives

$$
\mathbb{E}_{x} e^{-\int_{0}^{t} V(\omega(z)) d z}=1-V(x) t+\frac{t^{2}}{2}\left(V(x)^{2}-\Delta V(x)\right)+o\left(t^{2}\right)
$$
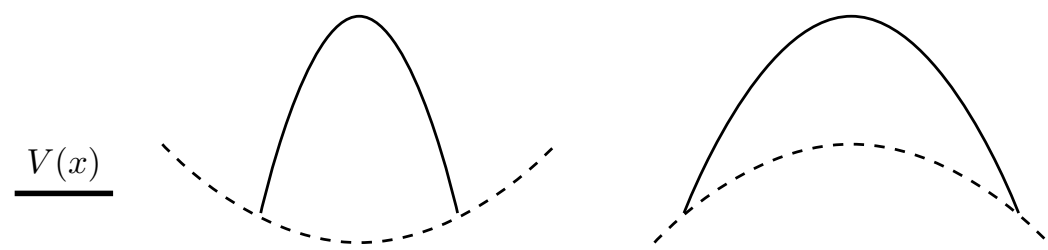

Figure 2. Two potentials (dashed) having comparable numerical value $\sim V(x)$. Local convexity enforces stronger decay of the eigenfunction, local concavity flatter decay. 
A geometric interpretation would be as follows: local convexity $(\Delta V(x)>0)$ yields stronger decay of the expectation and thus enforces a stronger decay of the eigenfunction; conversely, local concavity enforces slightly less decay (compared to flat potentials at the same numerical scale).

2.3. The case $V \leq \lambda$. The description above only discusses the cases, where locally $V \geq \lambda$ (in order to stress the similarity to Agmon's inequality), however, it is clear that a similar argument applies whenever $V \leq \lambda$. In that case the expectation over the path integrals will grow and this will imply variation at scale $\sqrt{t_{x}}$ where

$$
t_{x}=\inf \left\{t>0: \mathbb{E}_{x} e^{\lambda t-\int_{0}^{t} V(\omega(z)) d z} \geq 2\right\} .
$$

Presented differently, if $V \leq \lambda$ in such a way that there is local growth in $t$ of $\mathbb{E}_{x} e^{\lambda t-\int_{0}^{t} V(\omega(z)) d z}$, then the only way for

$$
\phi(x)=\mathbb{E}_{x}\left(\phi(\omega(t)) e^{\lambda t-\int_{0}^{t} V(\omega(z)) d z}\right)
$$

to be valid is for $\phi(\omega(t))$ to be, on average, smaller than $\phi(x)$ which indicates doubling of size on the length scale $\sqrt{t_{x}}$. In particular, the nonlocal formulation also applies to the setting where the classical $\sqrt{(V-\lambda)_{+}}$yields no more information. Using this formulation, we can recover the classical intuition for Laplacian eigenfunctions: if $-\Delta \psi=\lambda \psi$, then one expects $\psi$ to oscillate on the wavelength $\sim \lambda^{-1 / 2}$. In our case, if $V \ll \lambda$, then

$$
-\Delta \psi=(\lambda-V) \psi \sim \lambda \psi
$$

and the variation estimate guarantees oscillation on scale $\sqrt{\lambda} / c$, where $c \sim \lambda-V \sim \lambda$ and thus $\sqrt{\lambda} / c \sim \lambda^{-1 / 2}$. Another way of seeing this is that we expect a doubling on the scale $\sim \sqrt{t_{x}}$ and whenever $V \ll \lambda$, then

$$
t_{x}=\inf \left\{t>0: \mathbb{E}_{x} e^{\lambda t-\int_{0}^{t} V(\omega(z)) d z} \geq 2\right\} \sim \inf \left\{t>0: \mathbb{E}_{x} e^{\lambda t} \geq 2\right\} \sim \frac{1}{\lambda} .
$$

2.4. Landscape from variation. So far, we have only discussed the variation estimate; the relationship with landscapes is easily visualized: pick a sequence of points $x_{1}, x_{2}, \ldots, x_{k} \cdots \in \Omega$, compute $t_{x_{j}}$ and draw circles with radius $\sqrt{t_{x_{j}}}$ around the points. Smaller circles correspond to variation by a factor on a smaller spatial scale (i.e. faster growth/decay). The 'valleys' of the landscape correspond to regions with smaller circles (crossing them causes a variation by a factor 2 and thus frequent crossing is equivalent to either large growth or large decay) whereas 'hills' correspond to regions larger circles. This essentially reproduces the landscape generated by $u$ since both $\sqrt{t_{x}}$ and $u$ may be regarded as mollifications of $1 / V$.

The computation of $t_{x_{j}}$ is clearly nontrivial, however, up to first order it is easy, since

$$
\mathbb{E}_{x} e^{-\int_{0}^{t} V(B(\omega(z))) d z} \sim 1-\mathbb{E}_{x} \int_{0}^{t} V(B(\omega(z))) d z
$$

and, by linearity, we can exchange expectation and integration

$$
\begin{aligned}
\mathbb{E}_{x} \int_{0}^{t} V(B(\omega(z))) d z & =\int_{0}^{t} \mathbb{E}_{x} V(B(\omega(z))) d z \sim \int_{0}^{t}\left(e^{z \Delta} V\right)(x) d z \\
& =\left[\left(\int_{0}^{t} e^{z \Delta} d z\right) * V\right](x) .
\end{aligned}
$$


This is essentially accurate as long as $d(x, \partial \Omega) \gg \sqrt{t}$ while it overestimates the quantity as soon as $d(x, \partial \Omega) \lesssim \sqrt{t}$. In contrast, the landscape function $\lambda u(x)$ has a simpler and more convenient linear scaling in the eigenvalue $\lambda$. Indeed, $\lambda u(x)$ arises naturally from the following heuristic, which nicely clarifies how $u(x)$ interacts locally with the semigroup induced by $(-\Delta+V)$.

Heuristic. We compute an expansion of $e^{t(\Delta-V)} u(x)$ for $t$ small in two different ways. Note that

$$
(-\Delta+V) u=1 \quad \text { and thus, for } t \text { small, } \quad e^{t(\Delta-V)} u(x)=u(x)-t+o(t) .
$$

However, the semigroup may also be expanded using Feynman-Kac and for $t$ small

$$
\begin{aligned}
\left(e^{t(\Delta-V)} u\right)(x) & =\mathbb{E}_{x}\left(u(\omega(t)) e^{-\int_{0}^{t} V(\omega(z)) d z}\right) \\
& =\mathbb{E}_{x}\left(u(\omega(t))\left(1-\int_{0}^{t} V(\omega(z)) d z\right)\right) \\
& \sim u(x)-u(x) \mathbb{E}_{x} \int_{0}^{t} V(\omega(z)) d z .
\end{aligned}
$$

By matching the coefficient in the linear term, we get that

$$
u(x) \mathbb{E}_{x} \int_{0}^{t} V(\omega(z)) d z \sim t .
$$

Therefore, for $t$ sufficiently small

$$
\mathbb{E}_{x} \int_{0}^{t} V(\omega(z)) d z \sim \frac{t}{u(x)}
$$

and thus

$$
\mathbb{E}_{x} e^{\lambda t-\int_{0}^{t} V(\omega(z)) d z} \sim 1+\left(\lambda-\frac{1}{u(x)}\right) t .
$$

This heuristic naturally recovers decay in the region $\{x: 1 / u(x)>\lambda\}$. The only inaccuracy is that we actually have $\mathbb{E}_{x} u(\omega(t)) \sim u(x)+t \Delta u(x)$. Indeed, unless $|\Delta u(x)|$ is very large, we get from

$$
(-\Delta+V) u(x)=1 \quad \text { that } \quad u(x) \sim \frac{1}{V(x)} .
$$

In that case it is not very surprising that $1 / u(x)$ is effective at predicting decay. However, the relationship runs deeper than that: using again Feynman-Kac we get

$$
\begin{aligned}
e^{t(\Delta-V)} 1 & =\mathbb{E}_{x} e^{-\int_{0}^{t} V(\omega(z)) d z}, \\
e^{t(\Delta-V)^{-1}} 1 & =1-t u(x)+o(t) .
\end{aligned}
$$

Furthermore, information up to second order appears with the correct sign. We can rewrite $(-\Delta+V) u=1$ as

$$
u=\frac{1}{V}+\frac{\Delta u}{V} .
$$

Assuming $\Delta u \sim 0$ gave the first order approximation $u \sim 1 / V$. The next natural step is to iterate this

$$
u \sim \frac{1}{V}+\frac{\Delta\left(\frac{1}{V}\right)}{V}=\frac{1}{V}+2 \frac{|\nabla V|^{2}}{V^{3}}-\frac{\Delta V}{V^{3}} .
$$


Restricting to local extrema, we see that (up to lower order terms) $1 / u$ is larger than $V$ in local minima and smaller than $V$ in local maxima (thus recreating the behavior from above).

\section{NEW LANDSCAPE FUNCTIONS}

3.1. Nonlocal refinement. From now on, a 'landscape function' refers to any function $h(x)$ with the property that for a fixed eigenvalue $\lambda$ the eigenfunction $(-\Delta+V) \phi=\lambda \phi$ satisfies

$$
|\phi(x)| \leq h(x)\|\phi\|_{L^{\infty}} .
$$

$h(x)=1$ is trivially admissible. We will continue to use $u(x)$ to denote the classical landscape function given as the solution of $(-\Delta+V) u=1$. It satisfies the inequality with $h(x)=\lambda u(x)$.

Theorem 2 (Landscape bootstrapping). Suppose $(-\Delta+V) \phi=\lambda \phi$ and $h(x)$ satisfies

$$
|\phi(x)| \leq h(x)\|\phi\|_{L^{\infty}} ;
$$

then the same inequality holds for $h(x)$ replaced by

$$
\begin{aligned}
h_{1}(x) & =\inf _{t \geq 0} \mathbb{E}_{x}\left(h\left(\omega_{t}\right) e^{\lambda t-\int_{0}^{t} V(\omega(s)) d s}\right) \\
& =\inf _{t \geq 0} e^{\lambda t} e^{t(\Delta-V)} h(x) .
\end{aligned}
$$

By letting $t \rightarrow 0$, we get $h_{1}(x) \leq h(x)$. There is a delicate balance between two terms: $e^{t(\Delta-V)} h(x)$ is a diffusion semigroup inducing exponential decay which is counteracted by the exponential growth $e^{\lambda t}$. It is interesting to note that the function $\lambda u(x)$ plays a distinguished role and is characterized by the fact that no purely local (i.e. $t \rightarrow 0$ ) considerations have any effects.

Proposition. Suppose $f \in C^{2}(\Omega)$ satisfies

$$
\left.\frac{d}{d t} e^{\lambda t} e^{t(\Delta-V)} f(x)\right|_{t=0}=0, \quad \text { then } \quad f(x)=\lambda u(x) .
$$

Proof. The argument is immediate. Note that for $t$ small

$$
\begin{aligned}
e^{\lambda t} e^{t(\Delta-V)} f(x) & =\left(1+\lambda t+\mathcal{O}\left(t^{2}\right)\right)\left(1+t(\Delta-V) f(x)+\mathcal{O}\left(t^{2}\right)\right) \\
& =1+t\left(\lambda+(\Delta-V) f(x)+\mathcal{O}\left(t^{2}\right)\right)
\end{aligned}
$$

and therefore $f(x)=(-\Delta+V)^{-1} \lambda=\lambda u(x)$.

3.2. Computational tricks. The main results from the previous section allow for the generation of new landscape functions out of $\lambda u(x)$, however, one should consider that solving $e^{t(\Delta-V)} u(x)$ may be as hard or harder than directly computing eigenfunctions. The purpose of this section is to suggest a cheap way of creating computationally feasible improvements. The original proofs [11 demonstrating

$$
|\phi(x)| \leq \lambda u(x)\|\phi\|_{L^{\infty}}
$$

use Green's functions. A very simple argument that we could not find in the literature is as follows. 
Proof. $\phi$ is an eigenfunction and $-\Delta+V$ is an elliptic operator. The maximum principle yields

$$
\phi(x)=(-\Delta+V)^{-1} \lambda \phi(x) \leq(-\Delta+V)^{-1} \lambda\|\phi\|_{L^{\infty}}=\lambda\|\phi\|_{L^{\infty}}(-\Delta+V)^{-1} 1,
$$

where the last term is precisely $u(x)$.

The very simple proof immediately suggests two improvements. The first improvement would be to iterate the inequality: let $k \in \mathbb{N}$ be arbitrary. Then we have the inequality

$$
\phi(x)=(-\Delta+V)^{-k} \lambda^{k} \phi(x) \leq(-\Delta+V)^{-k} \lambda^{k}\|\phi\|_{L^{\infty}}=\lambda^{k}\|\phi\|_{L^{\infty}}(-\Delta+V)^{-k} 1 .
$$

This variant was already known to Filoche \& Mayboroda; its downside is that the increased power on the eigenvalue tends to make the bounds worse for higher eigenfunctions. There exists an elementary improvement that preserves linear scaling in the eigenvalue.

Proposition. If $(-\Delta+V) \phi=\lambda \phi$, then

$$
|\phi(x)| \leq\left(\lambda(-\Delta+V)^{-1} \min (\lambda u(x), 1)\right)\|\phi\|_{L^{\infty}} .
$$

Proof. We bootstrap the original inequality and have

$$
\phi=(-\Delta+V)^{-1} \lambda \phi \leq \lambda(-\Delta+V)^{-1}|\phi| \leq \lambda(-\Delta+V)^{-1} \min (\lambda u(x), 1)\|\phi\|_{L^{\infty}} .
$$

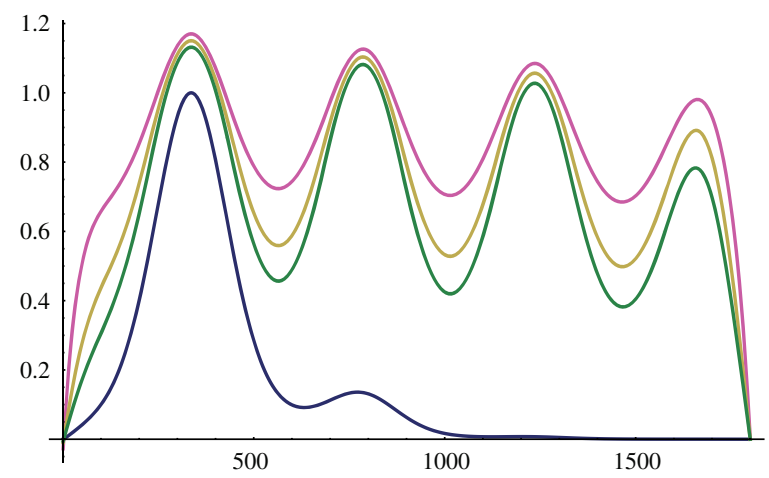

Figure 3. The profile $\phi_{1} /\left\|\phi_{1}\right\|_{L^{\infty}}$ (blue), the landscape function $u(x)$ (purple) and the first two refinements (yellow, green).

The reason why such a simple argument could indeed be effective is that the landscape function is sometimes larger than 1; in that case we can perform a simple cut-off at 1 and iterate to get additional information; alternatively, one could use the bound from the previous section in a neighborhood to propagate the gain from the cutoff to nearby regions. Clearly, that simple argument could also be iterated and, by the same token,

$$
|\phi(x)| \leq\left(\lambda(-\Delta+V)^{-1} \min \left(1, \lambda(-\Delta+V)^{-1} \min (\lambda u(x), 1)\right)\right)\|\phi\|_{L^{\infty}} .
$$

Like the original landscape function, these improvements can only be effective for $\lambda$ small: for $\lambda$ large, we will have $\lambda u(x) \geq 1$ everywhere except for small regions close to the boundary and thus $\min (\lambda u(x), 1) \sim 1$ and the argument will merely recreate $u$. 


\section{Proofs}

4.1. Proof of Theorem 1. The main idea has already been outlined above. We use

$$
\phi\left(x_{0}\right)=\mathbb{E}\left(\phi(\omega(t)) e^{\lambda t-\int_{0}^{t} V(\omega(z)) d z}\right)
$$

for a suitable time $t$. Almost all paths are contained within a ball of radius $\sim \sqrt{t}$ (suggesting to set $t=1 / c$ ). However, some Brownian motion paths will actually leave that ball and may give a large additional contribution but the likelihood of that happening is small. Using the standard reflection principle implies for the one-dimensional Brownian motion $B(t)$ that

$$
\mathbb{P}\left(\max _{0<s<t} B(s) \geq a\right)=2 \mathbb{P}(B(t) \geq a) .
$$

This implies that the likelihood of a Brownian motion leaving a ball of fixed radius for some time $0<s<t$ can be bounded by the likelihood of having left the ball at time $t$. More precisely, the standard Gaussian heat kernel estimate yields for some universal constants $c_{1}, c_{2}>0$ (depending only on the dimension) and fixed $t>0$

$$
\mathbb{P}\left(\|\omega(t)-\omega(0)\| \geq \delta t^{1 / 2}\right) \leq c_{1} e^{-c_{2} \delta^{2}} .
$$

This holds at a much greater level of generality [10]. The proof consists of showing that all the quantities work together as described by doing the algebra.

Proof. We assume that

$$
\forall x \in B: \frac{1}{2}\left|\phi\left(x_{0}\right)\right| \leq|\phi(x)| \leq 2\left|\phi\left(x_{0}\right)\right|
$$

with

$$
B=B\left(x_{0}, \frac{c_{3}}{\sqrt{c}} \sqrt{\frac{\lambda}{c}+\log \left(c_{3} \frac{\|\phi\|_{L^{\infty}}}{\left|\phi\left(x_{0}\right)\right|}\right)}\right) \subset \Omega,
$$

where $c_{3}$ is some constant we are allowed to choose depending on $c_{1}, c_{2}$ (which only depend on the dimension). We start with assuming that

$$
V-\lambda \geq c \quad \text { uniformly on } B \text {. }
$$

The case $V(x)-\lambda \leq-c$ is similar and will be described afterwards. We can assume without loss of generality that $\phi\left(x_{0}\right)>0$. The time scale of the argument will be $t=1 / c$. For a Brownian motion $\omega(s)$ started in $x_{0}$ we distinguish two cases:

- Case 1 (generic). $\{\omega(s): 0 \leq s \leq t\} \subset B$.

- Case 2 (rare). $\{\omega(s): 0 \leq s \leq t\} \not \subset B$.

Case 1 is very easy to deal with: in that case, we can easily bound any single path fully via

$$
\begin{aligned}
\phi(\omega(t)) e^{\lambda t-\int_{0}^{t} V(\omega(z)) d z} & \leq 2 \phi\left(x_{0}\right) e^{\int_{0}^{t}(\lambda-V(\omega(z))) d z} \\
& \leq 2 \phi\left(x_{0}\right) e^{-t c}
\end{aligned}
$$

and the same argument holds in expectation for all paths conditioned on Case 1. It remains to consider Case 2. If it occurs, then we only have the trivial bound (using $V \geq 0)$

$$
\phi(\omega(t)) e^{\lambda t-\int_{0}^{t} V(\omega(z)) d z} \leq\|\phi\|_{L^{\infty}} e^{\lambda t}
$$


which is large but the likelihood of that event is small. Combining this with the upper bound on the likelihood of Case 2 gives

$$
\phi\left(x_{0}\right)=\mathbb{E}_{x_{0}}\left(\phi(\omega(t)) e^{\lambda t-\int_{0}^{t} V(\omega(z)) d z}\right) \leq 2 e^{-t c} \phi\left(x_{0}\right)+c_{1} e^{-c_{2} \delta^{2}} e^{\lambda t}\|\phi\|_{L^{\infty}} .
$$

We will derive a contradiction to this inequality by setting by setting $t=1 / c$ and

$$
\delta=c_{3} \sqrt{\frac{\lambda}{c}+\log \left(c_{3} \frac{e^{\frac{\lambda}{c}}\|\phi\|_{L^{\infty}}}{\left|\phi\left(x_{0}\right)\right|}\right)} .
$$

Simple algebra allows us to reformulate the inequality as

$$
\phi\left(x_{0}\right) \leq 2 e^{-1} \phi\left(x_{0}\right)+\varepsilon_{c_{1}, c_{2}, c_{3}} \phi\left(x_{0}\right),
$$

where

$$
\varepsilon_{c_{1}, c_{2}, c_{3}}=\frac{c_{1}}{c_{3}^{c_{2} c_{3}^{2}}} e^{-\frac{\lambda}{c}\left(c_{2} c_{3}^{2}-1\right)}\left(\frac{\left|\phi\left(x_{0}\right)\right|}{\|\phi\|_{L^{\infty}}}\right)^{c_{2} c_{3}^{2}-1}
$$

can be made arbitrarily small by making $c_{3}$ sufficiently large (depending only on $\left.c_{1}, c_{2}\right)$. This gives a contradiction since $2 e^{-1}<1$ and concludes the first case. The other statement to consider is

$$
V-\lambda \leq-c \quad \text { uniformly on } B .
$$

Here, essentially all signs are reversed and we show that there is too much local growth. We can again distinguish Case 1 and Case 2 and get for Case 1 that

$$
\begin{aligned}
\phi(\omega(t)) e^{\lambda t-\int_{0}^{t} V(\omega(z)) d z} & \geq \frac{1}{2} \phi\left(x_{0}\right) e^{\int_{0}^{t}(\lambda-V(\omega(z))) d z} \\
& \geq \frac{1}{2} e^{t c} \phi\left(x_{0}\right) .
\end{aligned}
$$

The second case is again completely without control and we can only use the trivial estimate

$$
\phi(\omega(t)) e^{\lambda t-\int_{0}^{t} V(\omega(z)) d z} \geq-e^{\lambda t}\|\phi\|_{L^{\infty}} .
$$

Altogether, this yields

$$
\phi\left(x_{0}\right)=\mathbb{E}_{x_{0}}\left(\phi(\omega(t)) e^{\lambda t-\int_{0}^{t} V(\omega(z)) d z}\right) \geq \mathbb{P}(\text { Case } 1) \frac{1}{2} e^{t c} \phi\left(x_{0}\right)-c_{1} e^{-c_{2} \delta^{2}} e^{\lambda t}\|\phi\|_{L^{\infty}} .
$$

We set $t=100 / c$ and

$$
\delta=c_{3} \sqrt{\frac{\lambda}{c}+\log \left(c_{3} \frac{e^{\frac{\lambda}{c}}\|\phi\|_{L^{\infty}}}{\left|\phi\left(x_{0}\right)\right|}\right)} .
$$

We first note that

$$
\mathbb{P}(\text { Case } 1) \geq 1-c_{1} e^{-c_{2} \delta^{2}} .
$$

The same computation as above shows that

$$
c_{1} e^{-c_{2} \delta^{2}}=\frac{c_{1}}{c_{3}^{c_{2} c_{3}^{2}}} e^{-\frac{\lambda}{c}\left(c_{2} c_{3}^{2}\right)}\left(\frac{\left|\phi\left(x_{0}\right)\right|}{\|\phi\|_{L^{\infty}}}\right)^{c_{2} c_{3}^{2}} \leq \frac{c_{1}}{c_{3}^{c_{2} c_{3}^{2}}} e^{-\frac{\lambda}{c}\left(c_{2} c_{3}^{2}\right)},
$$

which can be made smaller than $1 / 10$ for $c_{3}$ sufficiently large (depending only on $\left.c_{1}, c_{2}\right)$. Thus, for $c_{3}$ sufficiently large,

$$
\phi\left(x_{0}\right)=\mathbb{E}_{x_{0}}\left(\phi(\omega(t)) e^{\lambda t-\int_{0}^{t} V(\omega(z)) d z}\right) \geq \frac{9}{10} e^{100} \phi\left(x_{0}\right)-c_{1} e^{-c_{2} \delta^{2}} e^{\lambda t}\|\phi\|_{L^{\infty}},
$$


where the second term satisfies

$$
\left|c_{1} e^{-c_{2} \delta^{2}} e^{\lambda t}\right|\|\phi\|_{L^{\infty}} \leq \frac{c_{1}}{c_{3}^{c_{2} c_{3}^{2}}} e^{-\frac{\lambda}{c}\left(c_{2} c_{3}^{2}-1\right)}\left(\frac{\left|\phi\left(x_{0}\right)\right|}{\|\phi\|_{L^{\infty}}}\right)^{c_{2} c_{3}^{2}-1} \phi\left(x_{0}\right)
$$

and can be made smaller than $\left|\phi\left(x_{0}\right)\right| / 2$ for $c_{3}$ sufficiently large (again depending only on $\left.c_{2}, c_{3}\right)$.

\subsection{Proof of Theorem 2.}

Proof. The proof used uses the identity for all $t \geq 0$ to introduce an infimum.

$$
\begin{aligned}
\phi(x)=\left(e^{\lambda t} e^{t(\Delta-V)} \phi\right)(x) & =\mathbb{E}_{x}\left(\phi\left(B_{t}(\omega)\right) e^{\lambda t-\int_{0}^{t} V(B(\omega(s))) d s}\right) \\
& =\inf _{t \geq 0} \mathbb{E}_{x}\left(\phi\left(B_{t}(\omega)\right) e^{\lambda t-\int_{0}^{t} V(B(\omega(s))) d s}\right) .
\end{aligned}
$$

By assumption $\phi$ is dominated by a landscape function $h(x)$ and thus

$$
\begin{aligned}
\inf _{t \geq 0} \mathbb{E}_{x}\left(\phi\left(B_{t}(\omega)\right) e^{\lambda t-\int_{0}^{t} V(B(\omega(s))) d s}\right) & \leq\|\phi\|_{L^{\infty}} \inf _{t \geq 0} \mathbb{E}_{x}\left(h\left(B_{t}(\omega)\right) e^{\lambda t-\int_{0}^{t} V(B(\omega(s))) d s}\right) \\
& =\|\phi\|_{L^{\infty}} \inf _{t \geq 0} e^{\lambda t} e^{t(\Delta-V)} h(x) .
\end{aligned}
$$

This concludes the argument.

4.3. Refined variation estimate. Since Brownian motion is isotropic, the proof of the variation estimate can cover a stronger result: simply put, the variation estimate is true because of curvature of the graph. More generally, we can show that if $h(x): \Omega \rightarrow \mathbb{R}$ is another function satisfying $h\left(x_{0}\right)=0$,

$$
\forall x \in B, \quad\left|e^{\lambda t} e^{t(\Delta-V)} h(x)\right| \leq \frac{1}{100} \phi(x),
$$

for the value of $t$ for which we wish to apply the variation estimate, then the function

$$
\phi(x)-h(x) \quad \text { doubles its sizes on } B \text {. }
$$

The best example is perhaps given by $V=0$ (eigenfunctions of the Laplace operator $-\Delta \phi=\lambda \phi)$ and $h(x)$ being the best linear approximation of $\phi$ in $x_{0}$

$$
h(x)=\left\langle\nabla \phi\left(x_{0}\right), x-x_{0}\right\rangle .
$$

This function is essentially invariant under heat flow for $d\left(x_{0}, \partial \Omega\right) \gg \sqrt{t}$ (with a negligible contribution from $\left|\nabla \phi\left(x_{0}\right)\right|$ that can be made precise). The variation estimate implies that $\phi(x)-h(x)$ doubles its size and since we have removed the tangent plane this implies that $\phi(x)-h(x)$ can't be too small because there is curvature in the graph. This also explains why the variation estimate does not apply when $V \sim \lambda$ : then the functions have no guaranteed curvature

$$
-\Delta \phi=(\lambda-V) \phi \sim 0
$$

and the doubling in size, if present at all, could possibly vanish once an affine function is removed.

\section{ACKNOWLEDGEMENTS}

The author is grateful to Ronald R. Coifman and Peter W. Jones for extensive discussions. The author was partially supported by an AMS-Simons travel grant and INET grant \#INO15-00038. 


\section{REFERENCES}

[1] Shmuel Agmon, Lectures on exponential decay of solutions of second-order elliptic equations: bounds on eigenfunctions of $N$-body Schrödinger operators, Mathematical Notes, vol. 29, Princeton University Press, Princeton, NJ; University of Tokyo Press, Tokyo, 1982. MR745286

[2] P. W. Anderson, Absence of Diffusion in Certain Random Lattices, Physical Review 109 (1958), 1492-1505.

[3] D. Arnold, G. David, D. Jerison, S. Mayboroda, and M. Filoche, The effective confining potential of quantum states in disordered media, ARXIV:1505.02684

[4] Laurent Bakri, Quantitative uniqueness for Schrödinger operator, Indiana Univ. Math. J. 61 (2012), no. 4, 1565-1580, DOI 10.1512/iumj.2012.61.4713. MR 3085618

[5] Catherine Bandle, Isoperimetric inequalities and applications, Monographs and Studies in Mathematics, vol. 7, Pitman (Advanced Publishing Program), Boston, Mass.-London, 1980. MR.572958

[6] Rodrigo Bañuelos and Tom Carroll, Brownian motion and the fundamental frequency of a drum, Duke Math. J. 75 (1994), no. 3, 575-602, DOI 10.1215/S0012-7094-94-07517-0. MR:1291697

[7] René Carmona, Pointwise bounds for Schrödinger eigenstates, Comm. Math. Phys. 62 (1978), no. 2, 97-106. MR505706

[8] R. Carmona and B. Simon, Pointwise bounds on eigenfunctions and wave packets in N-body quantum systems. V. Lower bounds and path integrals, Comm. Math. Phys. 80 (1981), no. 1, 59-98. MR623152

[9] René Carmona, Wen Chen Masters, and Barry Simon, Relativistic Schrödinger operators: asymptotic behavior of the eigenfunctions, J. Funct. Anal. 91 (1990), no. 1, 117-142, DOI 10.1016/0022-1236(90)90049-Q. MR1054115

[10] Xavier Fernique, Intégrabilité des vecteurs gaussiens (French), C. R. Acad. Sci. Paris Sér. A-B 270 (1970), A1698-A1699. MR0266263

[11] Marcel Filoche and Svitlana Mayboroda, Universal mechanism for Anderson and weak localization, Proc. Natl. Acad. Sci. USA 109 (2012), no. 37, 14761-14766, DOI 10.1073/pnas.1120432109. MR2990982

[12] Marcel Filoche and Svitlana Mayboroda, The landscape of Anderson localization in a disordered medium, Fractal geometry and dynamical systems in pure and applied mathematics. II. Fractals in applied mathematics, Contemp. Math., vol. 601, Amer. Math. Soc., Providence, RI, 2013, pp. 113-121, DOI 10.1090/conm/601/11916. MR3203829

[13] David Gilbarg and Neil S. Trudinger, Elliptic partial differential equations of second order, 2nd ed., Grundlehren der Mathematischen Wissenschaften [Fundamental Principles of Mathematical Sciences], vol. 224, Springer-Verlag, Berlin, 1983. MR.737190

[14] Steen Markvorsen and Vicente Palmer, Torsional rigidity of minimal submanifolds, Proc. London Math. Soc. (3) 93 (2006), no. 1, 253-272, DOI 10.1017/S0024611505015716. MR.2235949

[15] Barry Simon, Brownian motion, $L^{p}$ properties of Schrödinger operators and the localization of binding, J. Funct. Anal. 35 (1980), no. 2, 215-229, DOI 10.1016/0022-1236(80)90006-3. MR561987

[16] Stefan Steinerberger, Lower bounds on nodal sets of eigenfunctions via the heat flow, Comm. Partial Differential Equations 39 (2014), no. 12, 2240-2261, DOI 10.1080/03605302.2014.942739. MR3259555

[17] Michiel van den Berg, Large time asymptotics of the heat flow, Quart. J. Math. Oxford Ser. (2) 41 (1990), no. 162, 245-253, DOI 10.1093/qmath/41.2.245. MR.1053665

[18] Sijue Wu, Homogenization of differential operators, Acta Math. Appl. Sin. Engl. Ser. 18 (2002), no. 1, 9-14, DOI 10.1007/s102550200016. MR2010891

[19] Steve Zelditch, Local and global analysis of eigenfunctions on Riemannian manifolds, Handbook of geometric analysis. No. 1, Adv. Lect. Math. (ALM), vol. 7, Int. Press, Somerville, MA, 2008, pp. 545-658. MR2483375

Department of Mathematics, Yale University, 10 Hillhouse Avenue, New Haven, ConNECTICUT 06511

E-mail address: stefan.steinerberger@yale.edu 